\title{
Distribution and phylogeny of Wolbachia strains in wild mosquito populations in Sri Lanka
}

\author{
N. W. Nalaka P. Nugapola ${ }^{1,2}$, W. A. Priyanka P. De Silva ${ }^{1}$ and S.H.P. Parakrama Karunaratne ${ }^{1,3^{*}}$
}

\begin{abstract}
Background: Wolbachia are a group of maternally inherited intracellular bacteria known to be widespread among arthropods. Infections with Wolbachia cause declines of host populations, and also induce host resistance to a wide range of pathogens. Over the past few decades, researchers were curious to use Wolbachia as a biological tool to control mosquito vectors. During the present study, assessment of the prevalence of Wolbachia infections among wild mosquito populations in Sri Lanka where mosquito-borne diseases are a major health concern, was carried out for the first time. DNA was extracted from the abdomens of mosquitoes, collected from seven provinces, and screened for the presence of Wolbachia by PCR using wsp and groE primers. Group-specific and strain-specific primers were used to classify Wolbachia into the supergroups A and B, and into the strains Mel, AlbA and Pip.

Results: A total of 330 individual mosquitoes belonging to 22 species and 7 genera were screened. Eighty-seven mosquitoes (26.36\%) belonging to four species (i.e. Aedes albopictus, Culex quinquefasciatus, Armigeres subalbatus and Mansonia uniformis) were positive for Wolbachia infections. Primary vector of the dengue fever, Ae. aegypti was negative for Wolbachia infections while the secondary vector, Ae. albopictus, showed a very high infection rate. The filarial vector $C$. quinquefasciatus had a relatively high rate of infection. Japanese encephalitis vectors $C$. gelidus and C. triteaneorynchus, and the Anopheles vectors of malaria were negative for Wolbachia infections. Nine sequences of Wolbachia-positive PCR products were deposited in the GenBank and compared with other available data. Aedes albopictus was infected with both Wolbachia strains A (AlbA) and B (Pip) supergroups. Phylogenetic analysis of the wsp sequences showed two major branches confirming identities obtained from the PCR screening with strainspecific primers.
\end{abstract}

Conclusion: Wolbachia infections were found only among four mosquito species in Sri Lanka: Aedes albopictus, Culex quinquefasciatus, Armigeres subalbatus and Mansonia uniformis. Sequence data showed high haplotype diversity among the Wolbachia strains.

Keywords: Wolbachia strains, Phylogeny, Wsp, Biological control, Mosquito control, Sri Lanka

\section{Background}

Environment-friendly cost-effective strategies have emerged as crucial approaches in vector control programs today. The potential application of the symbiotic Wolbachia bacteria as a novel form of biological tool to control of mosquito-borne diseases, particularly dengue, has attracted an enormous attention during the past few decades. Wolbachia is a group of maternally inherited intracellular bacteria

\footnotetext{
*Correspondence: shppk@pdn.ac.lk; shppkaru@yahoo.com

'Department of Zoology, University of Peradeniya, Peradeniya 20400, Sri Lanka

${ }^{3}$ National Institute of Fundamental Studies, Hantana Road, Kandy 20000, Sri Lanka Full list of author information is available at the end of the article
}

which shows symbiotic relationships with arthropods and nematodes [1, 2]. They were first discovered by Hertig \& Wolbach [3] and since then the biology, diversity, distribution, evolution and the rate of host infection of Wolbachia have been studied widely $[1,2,4-7]$.

Infection with Wolbachia results in various reproductive abnormalities in the host including feminization, parthenogenesis, male killing and cytoplasmic incompatibility (CI) [1]. High infection rates, caused mainly through reproductive manipulations of the host, have enabled Wolbachia to spread rapidly within host populations making them a worthy candidate tool for 
consideration in vector control programs. Use of Wolbachia induced $\mathrm{CI}$ as a tool for mosquito control had been proposed in as early as 1960s [8] and few trials had been conducted in India in the 1970s [9]. Development of anti-Wolbachia based therapies to control mosquito borne diseases by replacement of vector populations and CI based incompatible insect techniques (IIT) are some of the recent approaches [10-13]. Successful control of the dengue vector mosquito Aedes aegypti by the introduction of Ae. aegypti infected with laboratory developed $w M e l$ and wMelPop strains of Wolbachia, to natural mosquito populations has been reported from the field trials conducted in Australia, Vietnam, Indonesia, Brazil and Colombia [14, 15]. Interestingly, the presence of Wolbachia induces host resistance to a wide range of pathogens including viruses, bacteria, protozoans and nematodes in trans-infected mosquitoes [13, 16-18]. Increased host resistance to pathogens is thought to be mainly through enhancing host immune responses, including haemolymph melanization, by Wolbachia and also through direct competition for cellular resources [13].

Wolbachia strains are globally distributed and currently considered as the most abundant endosymbionts found in invertebrates [19]. About 40\% of arthropod species are estimated to be infected with Wolbachia [20]. In arthropods, Wolbachia are believed to be primarily maternally transmitted within species [21] although horizontal transmission is also possible [22, 23]. Previous studies based on 16S rRNA gene sequences reported seven super-groups (super-groups A-H) of Wolbachia [2, 4]; supergroups $A$ and $B$ are the most common Wolbachia reported in arthropods [2]. Zhou et al. [5] reported that an improved phylogenetic resolution can be achieved by using the sequences of wsp gene which is evolving at a much faster rate than the other Wolbachia genes.

To our knowledge, the presence of Wolbachia in natural populations of mosquitoes has never been studied in Sri Lanka where mosquito-borne diseases are a major public health concern and more than 50,000 dengue cases have been reported among its population of 21 million people for the year 2016 [24]. Mosquito control programs of the country heavily rely on fogging operations using chemical insecticides [25] despite the increased vector resistance to insecticides and the adverse effect of insecticide fogging on non-target insects [26]. Environment-friendly new alternatives are urgently needed for vector control programs. The present study was undertaken to investigate the prevalence of Wolbachia infections among wild mosquito populations from different geographical regions of the country. Phylogenetic analysis of wsp sequences of the identified Wolbachia strains was also performed.

\section{Methods}

\section{Mosquito collection and identification}

Adults, larvae and eggs of different mosquito species were collected from seven provinces (i.e. Central, Sabaragamuwa, Western, Southern, Northern, North-western and Eastern) of Sri Lanka (Fig. 1). Adult collections were done using BG-Sentinel traps and CDC light traps. Larvae were collected using dippers and pipettes from mosquito breeding places. Mosquito eggs were collected using ovitraps. Larvae and eggs were reared to adults under laboratory conditions. Adult mosquitoes were identified using the standard taxonomic keys [27-30]. Identified specimens were labeled and stored at $-20{ }^{\circ} \mathrm{C}$ for later use.

\section{DNA extraction and polymerase chain reaction (PCR)}

DNA from the mosquito abdomens were extracted individually following the method described by Livak [31]. Five microliters from the extract was used for the PCR and double distilled water was used as the negative control. A known extract of Wolbachia-infected Ae. albopictus sample was used as the positive control.

Presence of Wolbachia was tested by amplifying Wolbachia surface protein gene using general wsp primers $[5,7,32]$, i.e. $w s p 81 \mathrm{~F}\left(5^{\prime}-\mathrm{TGG}\right.$ TCC AAT AAG TGA TGA AGA AAC-3') and wsp691R (5'-AAA AAT TAA ACG CTA CTC CA-3'). Resulting DNA fragments ranged from 590 to 632 bp depending on the Wolbachia strain. PCR amplifications were done in a thermal cycler (Techne-Flexigene, England) in $25 \mu \mathrm{l}$ reaction volumes. Each $25 \mu \mathrm{l}$ PCR reaction contained $7.5 \mu \mathrm{l} \mathrm{GoTaq}$ Green Master Mix (Promega, USA), $1.0 \mu$ l each from forward and reverse primers (Macrogen, Korea), $5 \mu \mathrm{l}$ DNA templates and $10.5 \mu \mathrm{l}$ nuclease free water (Promega, USA). The PCR cycling conditions were: initial denaturing at $95{ }^{\circ} \mathrm{C}$ for $3 \mathrm{~min}$, followed by 30 cycles of $94{ }^{\circ} \mathrm{C}$ for 1 min, $55{ }^{\circ} \mathrm{C}$ for $1 \mathrm{~min}$ and $72{ }^{\circ} \mathrm{C}$ for $1 \mathrm{~min}$. The final elongation was done at $72{ }^{\circ} \mathrm{C}$ for $10 \mathrm{~min}$, while the final holding was done at $4{ }^{\circ} \mathrm{C}$.

GroE primers, which amplify the $630 \mathrm{bp}$ region from the $g r o E$ operon [33], were also used to check for the presence of Wolbachia. PCR mixture composition was same as for $w s p$ primers and the PCR cycling conditions were: initial denaturing at $95{ }^{\circ} \mathrm{C}$ for $3 \mathrm{~min}$, followed by 30 cycles of $94{ }^{\circ} \mathrm{C}$ for $1 \mathrm{~min}, 48^{\circ} \mathrm{C}$ for $1 \mathrm{~min}$ and $72{ }^{\circ} \mathrm{C}$ for $1 \mathrm{~min}$, final elongation at $72{ }^{\circ} \mathrm{C}$ for $10 \mathrm{~min}$ and final holding at $4{ }^{\circ} \mathrm{C}$.

\section{Identification of Wolbachia groups and strains}

Samples positive for the $w s p$ primers were subjected to Wolbachia strain- and group-typing with wsp specific primers [5]. Group-specific primers were used to classify Wolbachia into supergroups A and B. Samples that were positive for supergroup A were screened with the Wolbachia strain-specific primers $\mathrm{Mel}$ and AlbA. Supergroup 


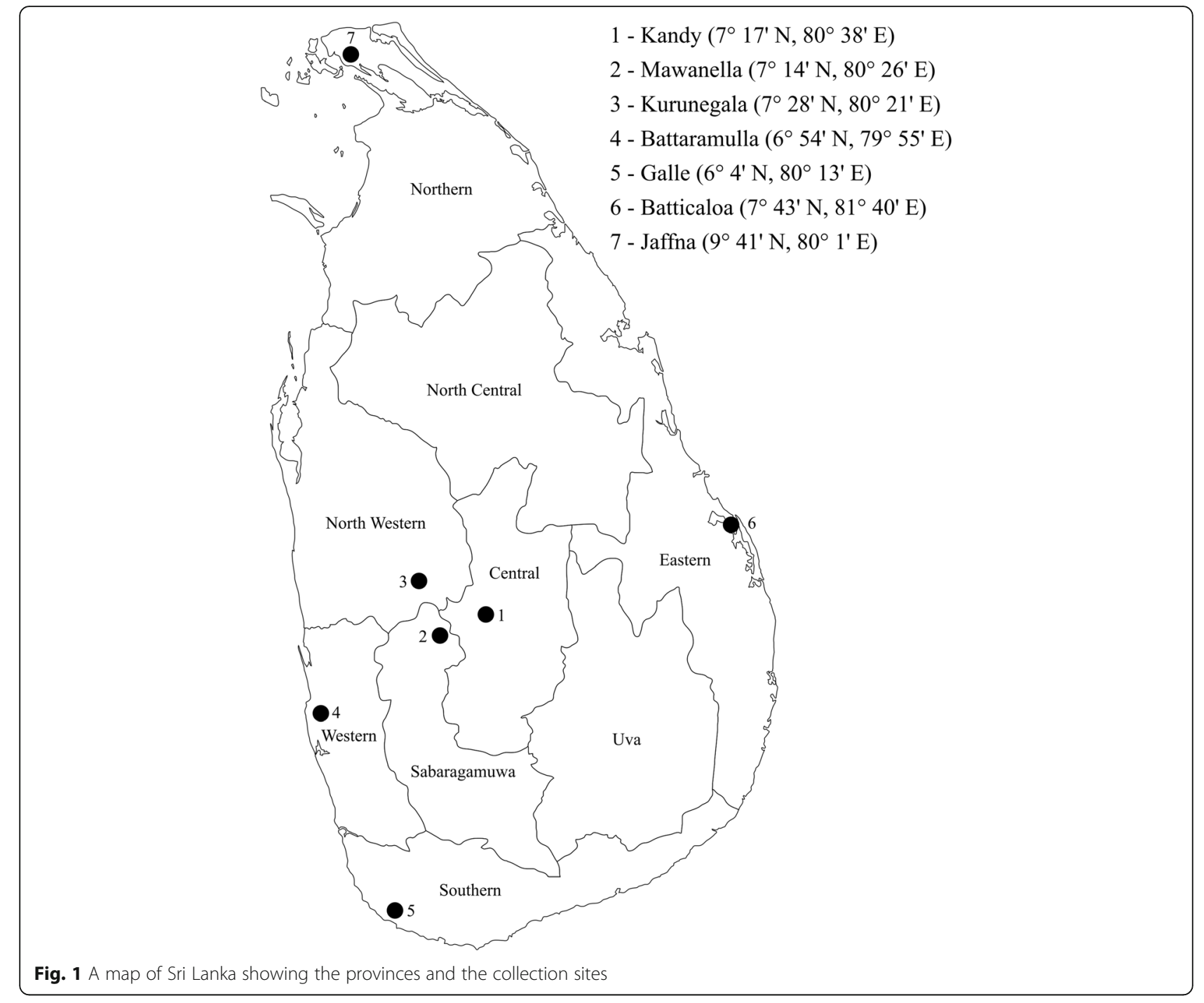

B samples were screened with Pip primer. Same PCR parameters described above were used. Five microliters of the PCR products were run on $1.5 \%$ agarose gel with a 100 bp standard DNA size marker (Invitrogen, California, USA) to confirm the PCR amplification. Samples with a DNA band of expected size were scored as positive.

\section{Wsp sequencing and phylogenetic analysis}

Nine Wolbachia-positive PCR products were directly sequenced using an automatic DNA sequencer (Applied Biosystems series 3500, USA). Sequence data were compared with the available data in the GenBank database using BLAST search and the sequences were deposited in the GenBank under accession numbers KY523666-KY523674.

Wsp sequences obtained from this study, reference wsp sequences obtained from EMBL alignment database [34] (accession no. DS32273) and wsp sequences obtained from GenBank [35] (KJ140127, AF020058,
KF725078, KF725079, KC668277, KC668275, KF725080, KC668284, KC668278, KJ140133, AY462864, GQ469982, KC137171, HM007832, DQ842453, HM007831) were edited and aligned in BioEdit 7.2.5 [36] using CustalW alignment algorithm [37]. Evolutionary history was inferred using the neighbor-joining (NJ) method [38] and the evolutionary distances were computed using the Kimura 2-parameter method [39]. Evolutionary analyses were conducted in MEGA7 [40].

\section{Results}

A total of 330 individual mosquitoes belonging to 22 species and 7 genera, collected from the 7 provinces of Sri Lanka, were individually screened for the presence of Wolbachia by PCR using wsp and groE primers. Presence of Wolbachia in different mosquito species from each province is given in the Table 1. A total of 87 mosquitoes, out of 330 (26.36\%), were positive for Wolbachia infection. Out of 22 species screened only 4 
Table 1 Prevalence of Wolbachia in mosquito species collected from different provinces of Sri Lanka

\begin{tabular}{|c|c|c|c|c|c|c|c|c|c|}
\hline \multirow[t]{2}{*}{ Mosquito species } & \multicolumn{7}{|c|}{ Province } & \multirow[t]{2}{*}{ Total } & \multirow[t]{2}{*}{$\%$ infectec } \\
\hline & $C$ & W & $\mathrm{E}$ & $\mathrm{N}$ & NW & SG & $S$ & & \\
\hline Aedes aegypti & $0 / 17$ & $0 / 2$ & $0 / 1$ & $0 / 10$ & $0 / 10$ & - & - & $0 / 40$ & 0.0 \\
\hline Aedes albopictus & $33 / 33$ & $1 / 29$ & - & - & $9 / 29$ & $10 / 10$ & $9 / 26$ & $62 / 127$ & 48.8 \\
\hline Aedes vittatus & - & - & - & - & $0 / 2$ & - & - & $0 / 2$ & 0.0 \\
\hline Anopheles species ${ }^{\mathrm{a}}$ & $0 / 1$ & - & $0 / 35$ & - & $0 / 46$ & - & - & $0 / 82$ & 0.0 \\
\hline Armigeres subalbatus & $4 / 4$ & - & - & - & & - & $3 / 3$ & $7 / 7$ & 100.0 \\
\hline Culex annulirostris & - & - & - & - & - & - & $0 / 2$ & $0 / 2$ & 0.0 \\
\hline Culex gelidus & - & - & $0 / 4$ & - & - & - & $0 / 2$ & $0 / 6$ & 0.0 \\
\hline Culex mimulus & - & - & - & - & $0 / 5$ & - & - & $0 / 5$ & 0.0 \\
\hline Culex quinquefasciatus & $5 / 17$ & - & $0 / 13$ & - & $8 / 17$ & - & $2 / 3$ & $15 / 50$ & 30.0 \\
\hline Culex triteaneorynchus & - & - & $0 / 2$ & - & - & - & $0 / 1$ & $0 / 3$ & 0.0 \\
\hline Mansonia uniformis & - & - & - & - & - & - & $3 / 3$ & $3 / 3$ & 100.0 \\
\hline Mimomia elegans & $0 / 1$ & - & - & - & - & - & - & $0 / 1$ & 0.0 \\
\hline Uranotaenia rutherfordi & $0 / 2$ & - & - & - & - & - & - & $0 / 2$ & 0.0 \\
\hline Total & $42 / 75$ & $1 / 31$ & $0 / 55$ & $0 / 10$ & $17 / 109$ & $10 / 10$ & $17 / 40$ & $87 / 330$ & 26.36 \\
\hline
\end{tabular}

Abbreviations: C Central, $W$ Western, S Southern, N Northern, E Eastern, SG Sabaragamuwa, NW North-Western

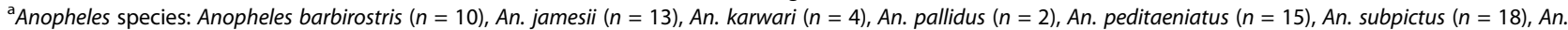
tessellatus $(n=6)$, An. varuna $(n=7)$, An. vegus $(n=7)$

species, i.e. Ae. albopictus, Culex quinquefasciatus, Armigeres subalbatus and Mansonia uniformis, were infected.

The primary vector of the dengue fever Ae. aegypti was negative $(n=40)$ for Wolbachia infection. The secondary vector of the dengue fever, Ae. albopictus, showed a $100 \%$ infection rate for the mosquitoes collected from Central $(n=33)$ and Sabaragamuwa $(n=$ 10) provinces followed by Southern $(34.6 \%$; 9 out of 26$)$ and North Western provinces (31.0\%; 9 out of 29). Only one Ae. albopictus was positive for Wolbachia from the Western province Pamples (3.4\%; 1 out of 29). Wolbachia infection rate was relatively high in the filarial vector $C$. quinquefasciatus; infection rates in this species from Southern, North-Western and Central provinces were 66.7, 47.1 and 29.4\%, respectively. However, C. quinquefasciatus collected from the Eastern Province $(n=13)$ were negative for Wolbachia infections. The Japanese encephalitis vectors $C$. gelidus $(n=6)$ and $C$. triteaneorynchus $(n=3)$ were not infected with Wolbachia. All of the Anopheles species tested ( $n=$ 82) were also negative for the Wolbachia infections. Infection rate of $M$. uniformis and Ar. subalbatus were $100 \%$ ( $n=3$ and $n=7$, respectively).

Results of the PCR performed with Wolbachia groupspecific wsp primers showed that Ae. albopictus $(n=10)$ is super-infected with Wolbachia strains belonging to A and B supergroups. Further screening using strain-specific $w s p$ primers revealed that the strain of Wolbachia in supergroup A is AlbA (379 bp band in agarose gel) and the strain in supergroup B is Pip (501 bp band in agarose gel) (Fig. 2).
Culex quinquefasciatus $(n=5)$ and M. uniformis (1 out of 2) were infected with Pip strain of Wolbachia which belongs to the supergroup B and Ar. subalbatus (2 out of 3) was infected with $A l b A$ strain (Table 2).

The results of the phylogenetic analysis of Wolbachia using $w s p$ sequences obtained from the present study and other 43 reference sequences are shown in Fig. 3. NJ tree was rooted between A and B super groups, and bootstrap values showed strong support for this separation. The phylogeny based on wsp sequences clearly showed that Wolbachia strains harbored by Ae. albopictus separated into $\mathrm{A}$ and $\mathrm{B}$ supergroups with strong bootstrap values. Of five Wolbachia sequences obtained from Ae. albopictus, three (KY523666, KY523667 and KY523670) were in super group $A$ and two (KY523668 and KY523669) were in super group B (Fig. 3). Wolbachia present in Ar. subalbatus (KY523671 and KY523672) were also clustered into Wolbachia supergroup A, but in a separate clade from Wolbachia present in Ae. albopictus mosquitoes. Wolbachia harbored by $C$. quinquefasciatus (KY523673) and $M$. uniformis (KY523674) were separated into Wolbachia supergroup B.

\section{Discussion}

To our knowledge, this is the first report to show the presence of Wolbachia bacteria in natural mosquito populations in Sri Lanka. The result of the PCR assays confirmed the previous findings that both wsp and groE primers are capable of identifying Wolbachia infections [7]. According to our results, the overall infection rate of all tested mosquitoes was $26.36 \%$. For natural mosquito 


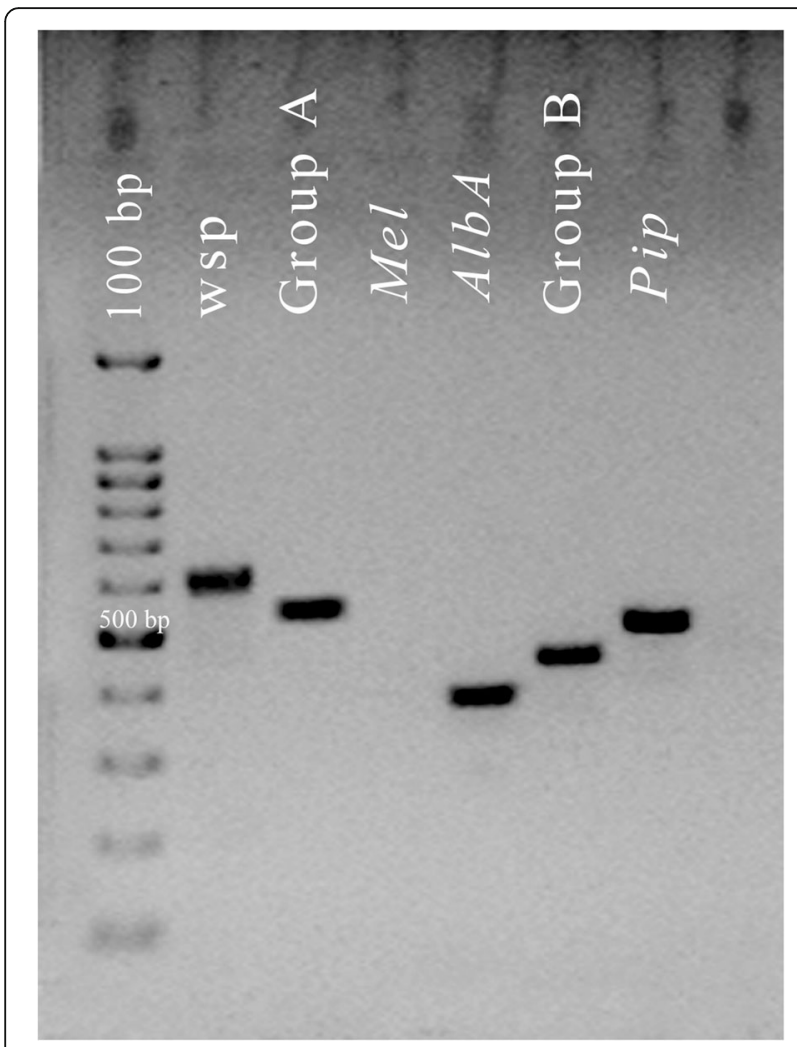

Fig. 2 Results of the Wolbachia strain identification PCR assays for Aedes albopictus mosquitoes collected from the central province of Sri Lanka $(n=33)$, using different primer sets: Lane 1: Wolbachia confirmation general primers wsp81F and wsp691R (600 bp band); Lane 2: Group A primers wsp136 and wsp691R (556 bp); Lane 3: Mel strain-specific primers wsp308F and wsp691R (405 bp); Lane 4: AlbA strain-specific primers wsp328F and 691R (379 bp); Lane 5: Group B primers wsp81F and wsp522R (442 bp); Lane 6: Pip strain-specific primers wspp183F and wsp 691R (501 bp). PCR products were electrophoresed in $1.5 \%$ agarose gel, stained with ethidium bromide and visualized under UV light populations, Wolbachia infection rates of 28.1 and $37.8 \%$ have been previously reported in Singapore [32] and Thailand [7], respectively.

In Sri Lanka, An. culicifacies is the major vector of malaria and An. subpictus is considered as a secondary vector. All of the other anopheline species tested have been incriminated as possible vectors of malaria [41]. Wolbachia could not be detected in any of the Anopheles species screened and this observation is consistent with previous reports [7, 32, 42]. Wolbachia-infected C. quinquefasciatus has been reported previously [43, 44]. Wolbachia was absent in the tested samples of the vectors of Japanese encephalitis, C. tritaeniorhynchus and C. gelidus; however, Wiwatanaratnabutr [7] had earlier encountered Wolbachia infections in both vectors. Kittayapong et al. [32] has reported that C. tritaeniorhynchus was positive for Wolbachia while C. gelidus was negative. The absence of Wolbachia infections in these vectors in Sri Lanka may be due to the small numbers of mosquitoes tested.

Although natural Wolbachia infections have never been reported from populations of the primary dengue vector Ae. aegypti, high infection rates have always been reported for Ae. albopictus mosquitoes [7, 15, 45]. For some reason infection rate in Ae. albopictus was remarkably low in the Western Province, i.e. one out of 29. During the present study, supergroup-typing of Wolbachia using PCR showed that all Ae. albopictus samples tested were co-infected with both A and B groups of Wolbachia. Specific primers derived from Wolbachia surface protein gene (wsp) have been used for Wolbachia strain identification and phylogeny analysis previously and were said to be the most sensitive for such an analysis [5]. The AB double infection of Wolbachia in Ae. albopictus at a rate of 99.4\% has been previously reported from Thailand $[32,45]$. It has been reported that multiple infections with several Wolbachia groups induce cytoplasmic incompatible phenotypes in female mosquitoes [1]. Further investigation

Table 2 Different groups and strains of Wolbachia present in the mosquito species Aedes albopictus ( $n=10)$, Culex quinquefasciatus $(n=5)$, Armigerus subalbatus $(n=4)$ and Mansonia uniformis $(n=2)$

\begin{tabular}{|c|c|c|c|c|c|c|c|}
\hline Wolbachia super-group & Strain & Primers $\left(5^{\prime}-3^{\prime}\right)$ & PCR product (bp) & $A A^{a}$ & $\mathrm{CQ}^{\mathrm{a}}$ & $A R^{a}$ & $M N^{b}$ \\
\hline \multirow[t]{3}{*}{ Group A } & & $\begin{array}{l}\text { 136 F: TGAAATTITAGCTCTITTC } \\
\text { 691R: AAAAATTAAACGCTACTCCA }\end{array}$ & 556 & 10 & - & 03 & - \\
\hline & Mel & $\begin{array}{l}308 \text { F: TTAAAGATGTAACATTTG } \\
\text { 691R: AAAAATTAAACGCTACTCCA }\end{array}$ & 405 & - & - & - & - \\
\hline & $A l b A$ & $\begin{array}{l}328 \text { F: CCAGCAGATACTATTGCG } \\
\text { 691R: AAAAATTAAACGCTACTCCA }\end{array}$ & 379 & 10 & - & 02 & - \\
\hline \multirow[t]{2}{*}{ Group B } & & $\begin{array}{l}81 \text { F: TGGTCCAATAAGTGATGAAGAAAC } \\
\text { 522R: ACCAGCTITGCTTGATA }\end{array}$ & 442 & 10 & 05 & - & 02 \\
\hline & Pip & $\begin{array}{l}183 \text { F: AAGGAACCGAAGTTCATG } \\
\text { 691R: AAAAATTAAACGCTACTCCA }\end{array}$ & 501 & 10 & 05 & - & 01 \\
\hline
\end{tabular}

${ }^{\mathrm{a}}$ From Central Province

${ }^{\mathrm{b}}$ From Southern Province

Abbreviations: AA Aedes albopictus, CQ Culex quinquefasciatus, AR Armigerus subalbatus, MN Mansonia uniformis 


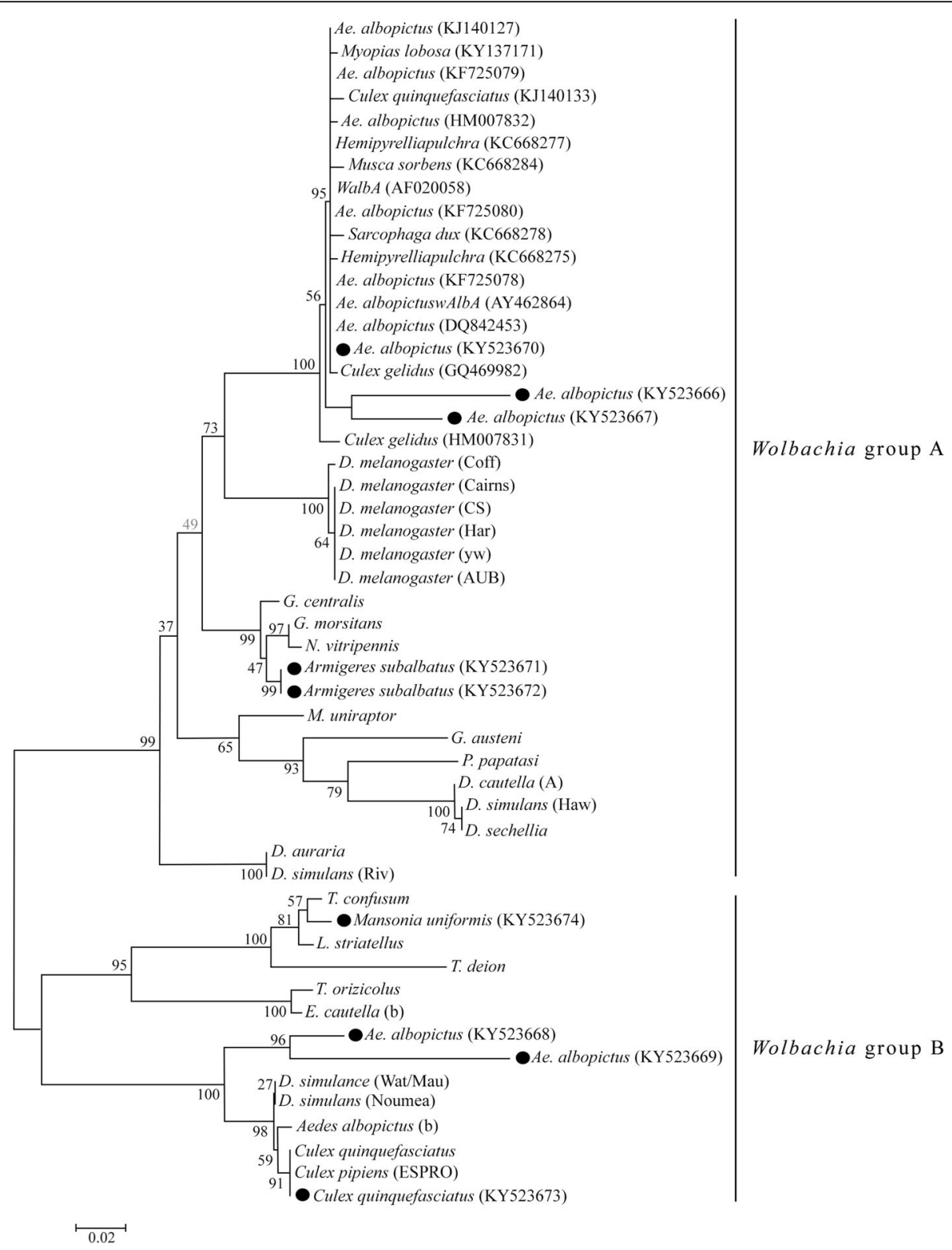

Fig. 3 Neighbor-joining tree generated from aligned wsp sequences. Tree shown is midpoint rooted and bootstrap values (1,000 replicates) are labelled next to the branches. Taxa are labelled as the host names from which the Wolbachia strains were obtained. Wsp sequences from the present study are marked with black dots

of Wolbachia groups into strains revealed that all Ae. albopictus samples tested were commonly infected with AlbA (group A) and Pip (group B) strains of Wolbachia. Kittayapong et al. [45] reported the same combination of strains of Wolbachia (AlbA and Pip) in Ae. albopictus, Ae. pseudoalbopictus and other Ae. (Stegomyia) spp. They reported that three out of five species of the subgenus Stegomyia were infected with both AlbA and Pip strains of Wolbachia. Our results also revealed the presence of the strain Pip in C. quinquefasciatus and M. uniformis, and the strain AlbA in Ar. subalbatus.
The identities obtained from the PCR screening with strain-specific primers were further confirmed during the phylogenetic analysis of the wsp sequences. It is evident that haplotype diversity of Wolbachia strains was very high. According to the NJ tree generated, three wsp sequences (Ae. albopictus: KY523666, KY523667 and KY523670) were clustered with a clade where Wolbachia AlbA sequences (AF020058 and AY462864) clustered together. Other sequences (Ae. albopictus: KY523668, KY523669; Culex quinquefasciatus: KY523673; Mansonia uniformis: KY523674) were in the 
Group B branch of the phylogenetic tree where wsp sequences of Wolbachia Pip strains from Cx. pipiens, Cx. quinquefasciatus, Drosophila simulans and Ae. albopictus (b) are found (Fig. 2).

Incidence of dengue is increasing at an alarming rate in Sri Lanka and other tropical countries. Control of dengue vector larvae with the common strategies such as spraying synthetic insecticides/Bacillus thuringiensis is not effective as the breeding sites of dengue vectors are mainly discarded receptacles and leaf axels among the vegetation [46]. Although the clearance of breeding sites is effective to a certain extent, elimination of vegetation is not possible. Efficacy of space spraying of adulticides is also questionable considering the actual effect on the mosquitoes and the extent of damage it causes to the non-target insects [26]. There is an urgent need to adopt alternative techniques such as Wolbachiainfected replacement [11-13], sterile male technique and radiation to combat the dengue and other vector populations in endemic areas [47-50]. The widespread distribution and various reproductive manipulations of Wolbachia have drawn attention of researchers to use Wolbachia as a biological control method. This approach is attempted by many researchers around the world and the field trials have demonstrated promising outcomes [10, 13-15].

Given the fact that Wolbachia reduces the life span of the host preventing the virus to complete its proper incubation period and also causes increased host immunity resisting the viral development in the host, Wolbachia would be a better choice for vector control leading to a large-scale reduction of the disease transmission. The present study is an initial attempt to produce basic information regarding the extent of natural infections of Wolbachia in wild mosquito populations in Sri Lanka. Although it is not found among wild Ae. aegypti populations, trans-infected Ae. aegypti has been successfully used in field trials [14, 15, 51-53]. Trans-infected mosquito lines may also be developed to combat diseases such as malaria and Japanese encephalitis. The present study, therefore provides important information to develop novel approaches in using Wolbachia to mosquito control, particularly in controlling mosquito populations that do not respond to conventional vector control strategies.

\section{Conclusions}

Wolbachia infections were found only among four mosquito species in Sri Lanka: Aedes albopictus, Culex quinquefasciatus, Armigeres subalbatus and Mansonia uniformis. Sequence data showed high haplotype diversity among the Wolbachia strains. Ae. albopictus samples were commonly infected with AlbA (group A) and Pip (group B). Pip was also found in C. quinquefasciatus and M. uniformis. Wolbachia strain AlbA was found in Ar. subalbatus.

\section{Acknowledgements}

Prof. SN Surendran (University of Jaffna, Sri Lanka), Dr. Devika Perera and Mr. MACM Mansoor (Anti Malaria Campaign, Kurunegala, Sri Lanka) are acknowledged for the assistance given during mosquito collection.

\section{Funding}

This study was funded by National Research Council, Sri Lanka (Grant No. 14-94).

\section{Availability of data and materials}

The datasets used and/or analysed during the current study are available from the corresponding author on reasonable request. The sequences were submitted to the GenBank database under accession numbers KY523666-KY523674.

\section{Authors' contributions}

SHPPK and WAPP De S conceptualized and designed the work. NWNPN collected data. All contributed to the analysis of data, discussion and writing the manuscript. All authors read and approved the final manuscript.

\section{Competing interests}

The authors declare that they have no competing interests.

Consent for publication

Not applicable.

Ethics approval and consent to participate

Not applicable.

\section{Publisher's Note}

Springer Nature remains neutral with regard to jurisdictional claims in published maps and institutional affiliations.

\section{Author details}

'Department of Zoology, University of Peradeniya, Peradeniya 20400, Sri Lanka. ${ }^{2}$ Postgraduate Institute of Science, University of Peradeniya, Peradeniya 20400, Sri Lanka. ${ }^{3}$ National Institute of Fundamental Studies, Hantana Road, Kandy 20000, Sri Lanka.

Received: 12 February 2017 Accepted: 3 May 2017

Published online: 10 May 2017

\section{References}

1. Werren JH. Biology of Wolbachia. Annu Rev Entomol. 1997:42:587-609.

2. Werren JH, Baldo L, Clark ME. Wolbachia: master manipulators of invertebrate biology. Nat Rev Microbiol. 2008;6:741-51.

3. Hertig M, Wolbach SB. Studies on Rickettsia-Like Micro-Organisms in Insects. J Med Res. 1924;44:329-74.

4. O'Neill SL, Giordano R, Colbert AM, Karr TL, Robertson HM. 165 rRNA phylogenetic analysis of the bacterial endosymbionts associated with cytoplasmic incompatibility in insects. Proc Natl Acad Sci USA. 1992;89: 2699-702.

5. Zhou W, Rousset F, O'Neill S. Phylogeny and PCR-based classification of Wolbachia strains using wsp gene sequences. Proc R Soc B Biol Sci. 1998; 265:509-15.

6. Hilgenboecker $\mathrm{K}$, Hammerstein $\mathrm{P}$, Schlattmann $\mathrm{P}$, Telschow $\mathrm{A}$, Werren JH. How many species are infected with Wolbachia?-A statistical analysis of current data. FEMS Microbiol Lett. 2008;281:215-20.

7. Wiwatanaratanabutr I. Geographic distribution of wolbachial infections in mosquitoes from Thailand. J Invertebr Pathol. 2013:114:337-40.

8. Laven $\mathrm{H}$. Eradication of Culex pipiens fatigans through cytoplasmic incompatibility. Nature. 1967;216:383-4.

9. Curtis CF, Adak T. Population replacement in Culex fatigens by means of cytoplasmic incompatibility. Laboratory experiments with non-overlapping generations. Bull World Health Organ. 1974;51:249-55.

10. Jeffries $\mathrm{CL}$, Walker $\mathrm{T}$. The potential use of Wolbachia-based mosquito biocontrol strategies for Japanese encephalitis. PLoS Negl Trop Dis. 2015:9: e0003576. 
11. Lambrechts L, Ferguson NM, Harris E, Holmes EC, McGraw EA, O’Neill SL, et al. Assessing the epidemiological effect of Wolbachia for dengue control. Lancet Infect Dis. 2015;15:862-6.

12. Brelsfoard CL, Dobson SL. An update on the utility of Wolbachia for controlling insect vectors and disease transmission. Asia-Pacific J Mol Biol Biotechnol. 2011;19:85-92.

13. Iturbe-Ormaetxe I, Walker T, O' Neill SL. Wolbachia and the biological control of mosquito-borne disease. EMBO Rep. 2011;12:508-18.

14. Nguyen TH, Le Nguyen H, Nguyen TY, Vu SN, Tran ND, Le TN, et al. Field evaluation of the establishment potential of wmel pop Wolbachia in Australia and Vietnam for dengue control. Parasit Vectors. 2015;8:563.

15. Ritchie S. Rear and release: a new paradigm for dengue control. Austral Entomol. 2014:53:363-7.

16. Glaser RL, Meola MA. The native Wolbachia endosymbionts of Drosophila melanogaster and Culex quinquefasciatus increase host resistance to West Nile virus infection. PLoS One. 2010;5:e11977.

17. Kambris Z, Cook PE, Phuc HK, Sinkins SP. Immune activation by lifeshortening Wolbachia and reduced filarial competence in mosquitoes. Science. 2009;326:134-6.

18. Moreira LA, Iturbe-Ormaetxe I, Jeffery JA, Lu G, Pyke AT, Hedges LM, et al. A Wolbachia symbiont in Aedes aegypti limits infection with dengue, chikungunya, and Plasmodium. Cell. 2009;139:1268-78.

19. Werren JH, Windsor DM. Wolbachia infection frequencies in insects: evidence of a global equilibrium? Proc R Soc B Biol Sci. 2000;267:1277-85.

20. Zug R, Hammerstein P. Still a host of hosts for Wolbachia: analysis of recent data suggests that $40 \%$ of terrestrial arthropod species are infected. PLoS One. 2012;7:e38544.

21. Skinner SW. Maternally inherited sex ratio in the parasitoid wasp Nasonia vitripennis. Science. 1982;215:1133-4

22. Schilthuizen MO, Stouthamer R. Horizontal transmission of parthenogenesisinducing microbes in Trichogramma wasps. Proc R Soc B Biol Sci. 1997;264: 361-6.

23. Werren JH, Zhang W, Guo LR. Evolution and phylogeny of Wolbachia: reproductive parasites of arthropods. Proc R Soc London B Biol Sci. 1995; 261:55-71.

24. Unit E. Distribution of notification (H399) dengue cases by month. Sri Lanka: Ministry of Health; 2016. http://www.epid.gov.lk/web/index.php?option=com_ casesanddeaths\&ltemid=448\&lang=en. Accessed 26 Dec 2016.

25. Karunaratne SHPP, Weeraratne TC, Perera MDB, Surendran SN. Insecticide resistance, and efficacy of space spraying and larviciding in the control of dengue vectors Aedes aegypti and Aedes albopictus in Sri Lanka. Pestic Biochem Physiol. 2013;107:98-105.

26. Abeyasuriya KGTN, Nugapola NWNP, Perera MDB, Karunaratne WAIP, Karunaratne SHPP. Effect of dengue mosquito control insecticide thermal fogging on non-target insects. Int J Trop Insect Sci. 2016;26:1-8.

27. Amarasinghe F. Illustrated keys to the genera of mosquitoes (Diptera: Culicidae) in Sri Lanka. J Natn Sci Coun Sri Lanka. 1995:23:183-211.

28. Amarasinghe F. Keys for the identification of the adults of genus Culex (Diptera: Culicidae) in Sri Lanka. J Natn Sci Coun Sri Lanka. 1995;23:221-39.

29. Reuben R, Tewari SC, Hiriyan J, Akiyama J. Illustrated keys to species of Culex (Culex) associated with Japanese encephalitis in Southeast Asia (Diptera: Culicidae). Mosq Syst. 1994;26:75-96.

30. Amarasinghe F. A guide to the identification of the anopheline mosquitoes (Diptera: culicidae) of Sri Lanka. I. Adult Females. Ceylon J Sci (Bio Sci). 1990; 21:1-16

31. Livak KJ. Organization and mapping of a sequence on the Drosophila melanogaster $X$ and $Y$ chromosomes that is transcribed during spermatogenesis. Genetics. 1984;107:611-34

32. Kittayapong P, Baisley KJ, Baimai V, O'Neill SL. Distribution and diversity of Wolbachia infections in Southeast Asian mosquitoes (Diptera: Culicidae). J Med Entomol. 2000;37:340-5.

33. Wiwatanaratanabutr I, Kittayapong P, Caubet Y, Bouchon D. Molecular phylogeny of Wolbachia strains in arthropod hosts based on groEhomologous gene sequences. Zoolog Sci. 2009;26:171-7.

34. Sterk P, Kulikova T, Kersey P, Apweiler R. The EMBL nucleotide sequence and genome reviews databases. Methods Mol Biol. 2007;406:1-21.

35. GenBank. https://www.ncbi.n/m.nih.gov/genbank/. Accessed 26 Dec 2016.

36. Hall T. Symposium on RNA Biology. III. RNA, tool and target. Research triangle park, North Carolina, USA. October 15-17, 1999. Proceed Nucleic Acids Symp Ser. 1999;41:95-8.
37. Thompson JD, Higgins DG, Gibson TJ. CLUSTAL W: improving the sensitivity of progressive multiple sequence alignment through sequence weighting, position-specific gap penalties and weight matrix choice. Nucleic Acids Res. 1994:22:4673-80.

38. Saitou N, Nei M. The neighbor-joining method: a new method for reconstructing phylogenetic trees. Mol Biol Evol. 1987:4:406-25.

39. Kimura M. A simple method for estimating evolutionary rates of base substitutions through comparative studies of nucleotide sequences. J Mol Evol. 1980:16:111-20.

40. Kumar S, Stecher G, Tamura K. MEGA7: Molecular Evolutionary Genetics Analysis version 7.0 for bigger datasets. Mol Biol Evol. 2016:33:1870-4.

41. Amerasinghe PH, Amerasinghe FP, Konradsen F, Fonseka KT, Wirtz RA. Malaria vectors in a traditional dry zone village in Sri Lanka. Am J Trop Med Hyg. 1999;60:421-9.

42. de Oliveira CD, Gonçalves DS, Baton LA, Shimabukuro PHF, Carvalho FD, Moreira LA. Broader prevalence of Wolbachia in insects including potential human disease vectors. Bull Entomol Res. 2015;105:305-15.

43. Osei-Poku J, Han C, Mbogo CM, Jiggins FM. Identification of Wolbachia strains in mosquito disease vectors. PLoS One. 2012;7:e49922.

44. Muniaraj M, Paramasivan R, Sunish IP, Arunachalam N, Mariappan T, Jerald Leo SV, et al. Detection of Wolbachia endobacteria in Culex quinquefasciatus by Gimenez staining and confirmation by PCR. J Vector Borne Dis. 2012;49: 258-61

45. Kittayapong P, Baimai V, O'Neill SL. Field prevalence of Wolbachia in the mosquito vector Aedes albopictus. Am J Trop Med Hyg. 2002;66:108-11.

46. Weeraratne TC, Perera MD B, Mansoor MACM, Karunaratne SHPP. Prevalence and breeding habitats of the dengue vectors Aedes aegypti and Aedes albopictus (Diptera: Culicidae) in the semi-urban areas of two different climatic zones in Sri Lanka. Int J Trop Insect Sci. 2013;33:216-26.

47. Yamada H, Parker AG, Oliva CF, Balestrino F, Gilles JRL. X-Ray-induced sterility in Aedes albopictus (Diptera: Culicidae) and male longevity following irradiation. J Med Entomol. 2014;51:811-6.

48. Alphey L, Benedict M, Bellini R, Clark GG, Dame DA, Service MW, et al. Sterile-insect methods for control of mosquito-borne diseases: an analysis. Vector Borne Zoonotic Dis. 2010;10:295-311.

49. Helinski ME, Parker AG, Knols BG. Radiation-induced sterility for pupal and adult stages of the malaria mosquito Anopheles arabiensis. Malar J. 2006:5:41.

50. FAO/IAEA. Sterile-male technique for eradication or control of harmful insects. In: Proceedings of a panel, Vienna. Vienna: Internationa Atomic Energy Agency. p. 137

51. Dutra HLC, dos Santos LMB, Caragata EP, Silva JBL, Villela DAM, Maciel-deFreitas $\mathrm{R}$, et al. From lab to field: The influence of urban landscapes on the invasive potential of Wolbachia in Brazilian Aedes aegypti mosquitoes. PLoS Negl Trop Dis. 2015:9:e0003689.

52. Yeap H, Axford JK, Popovici J, Endersby NM, Iturbe-Ormaetxe I, Ritchie SA, et al. Assessing quality of life-shortening Wolbachia-infected Aedes aegypti mosquitoes in the field based on capture rates and morphometric assessments. Parasit Vectors. 2014;7:58.

53. Hoffmann AA, Iturbe-Ormaetxe I, Callahan AG, Phillips BL, Billington K, Axford JK, et al. Stability of the wMel Wolbachia infection following invasion into Aedes aegypti populations. PLoS Negl Trop Dis. 2014;8:e3115.

\section{Submit your next manuscript to BioMed Central and we will help you at every step:}

- We accept pre-submission inquiries

- Our selector tool helps you to find the most relevant journal

- We provide round the clock customer support

- Convenient online submission

- Thorough peer review

- Inclusion in PubMed and all major indexing services

- Maximum visibility for your research

Submit your manuscript at www.biomedcentral.com/submit 